\title{
Editorial: Urban Vibrations \& Their Effects Upon Built Heritage: Measurements, Characterization, \& Simulations
}

\author{
Michele Palermo ${ }^{1 *}$, Simonetta Baraccani ${ }^{1}$, Maria Girardi ${ }^{2}$, Daniele Pellegrini ${ }^{2}$ and \\ Riccardo M. Azzara $^{3}$ \\ ${ }^{1}$ Department of Civil, Chemical, Environmental and Materials Engineering, University of Bologna, Bologna, Italy, ${ }^{2}$ Consiglio \\ Nazionale delle Ricerche, Istituto di Scienza e Tecnologie dell'Informazione "Alessandro Faedo", Pisa, Italy, ${ }^{3}$ Osservatorio \\ Sismologico di Arezzo, Istituto Nazionale di Geofisica e Vulcanologia, Arezzo, Italy
}

Keywords: dynamic identification, structural health monitoring, cultural heritage, ambient vibrations, environmental effects, operational modal analysis, finite element analysis, model updating

\section{Editorial on the Research Topic}

Urban Vibrations \& Their Effects Upon Built Heritage: Measurements, Characterization, \& Simulations

The numerous sources of vibration that can be recorded in the urban environment both at ground or at the top level of buildings represent a complex and largely unexplored field, whose interpretation could provide valuable information about the buildings' structural behavior. Urban vibrations may excite constructions in many different ways, generating discomfort, structural damage, and fatigue-related issues affecting their structural health. The characterization of such urban vibrations, and their use for dynamic identification and structural health monitoring, launched an emerging, new cross-disciplinary field known as urban seismology, which engages seismologists, structural engineers, and numerical analysts. The research contributions collected in this special issue deal with this emerging topic.

The work by D'Alessandro et al. summarizes the recent research carried out by the National Earthquake Observatory, Istituto Nazionale di Geofisica e Vulcanologia (ONT-INGV, Italy) related to the reduction of seismic risk through the implementation of urban-scale and building-scale monitoring networks. The work illustrates the different approaches, experiences and potential capabilities of urban seismic networks, structural health, and cultural heritage monitoring systems implemented in Italy by the ONT-INGV to achieve an effective integrated multi-scale system.

Assessing the effects of urban vibrations on historical buildings is of fundamental importance for their effective long-term conservation. In this regard, the work by Baraccani et al. investigated the effects of traffic-induced urban vibrations on the two Towers of Bologna, located in a highly congested area and particularly prone to fatigue-related damages. Seismometric measurement devices (tri-axial velocimeters) allowed the identification of several different vibration sources. The analyses use a large dataset of time records acquired from dynamic monitoring campaigns conducted during the last 10 years. Frequency-domain techniques and finite element models have been employed to evaluate the Towers' main dynamic properties and their variations with environmental effects. Time-domain analysis allowed the authors to quantify the amplitudes of traffic-induced vibrations.

Within the same context, Azzara et al. investigated the long-term dynamic behavior of two medieval masonry towers located in the old town of Lucca, namely the San Frediano Cathedral bell 
tower and the Clock Tower. The combined use of triaxial velocimeters and tri-axial accelerometers, developed for gravitation measurements, enabled to cover a wide range of frequencies and to measure the low-frequency displacements of the towers. Operational modal analysis and advanced finite element modeling techniques allowed to evaluate the effects of temperature variations on the towers' dynamic properties.

Operational modal analysis is also used in Lacanna et al. to assess the dynamic properties of the Baptistery of San Giovanni in Florence. Indeed, the output-only modal test represents a valuable alternative to forced vibration techniques when historical structures are investigated. This method takes advantage of natural sources of vibration without the need for artificially shaking the structure. The dynamic properties of the Baptistery are evaluated by means of the Enhanced Frequency Domain Decomposition technique. The modal parameters are then used to calibrate a finite element model by using a genetic algorithm. This procedure allows to obtain an accurate and robust model capable of reproducing the structure's dynamic behavior by minimizing the uncertainties related to the knowledge of the mechanical parameters.

Although urban seismology is still at its infancy, the research articles collected in this Special Issue demonstrate that the combined use of advanced dynamic monitoring techniques proper of seismology with numerical methods proper of structural dynamics may represent an effective tool for a comprehensive assessment of the structural health of the built environment in general and, more specifically, for long-term conservation of historical and monumental constructions.

\section{AUTHOR CONTRIBUTIONS}

MP prepared the draft. SB, MG, DP, and RA edited and reviewed the draft. All authors contributed to the article and approved the submitted version.

Conflict of Interest: The authors declare that the research was conducted in the absence of any commercial or financial relationships that could be construed as a potential conflict of interest.

Copyright (C) 2020 Palermo, Baraccani, Girardi, Pellegrini and Azzara. This is an open-access article distributed under the terms of the Creative Commons Attribution License (CC BY). The use, distribution or reproduction in other forums is permitted, provided the original author(s) and the copyright owner(s) are credited and that the original publication in this journal is cited, in accordance with accepted academic practice. No use, distribution or reproduction is permitted which does not comply with these terms. 